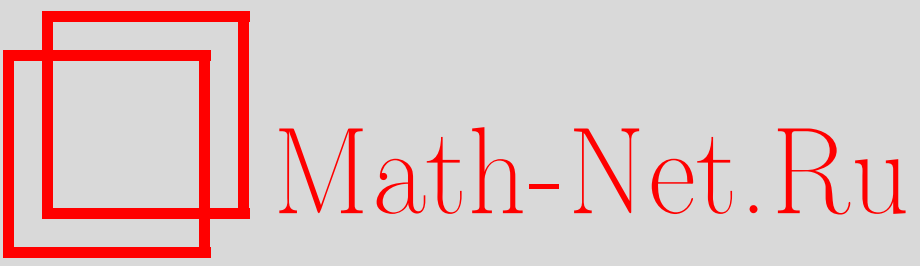

А. Р. Кессель, Р. Р. Нигматуллин, А. А. Хамзин, Н. А. Яковлева, Некоторые точные решения для модели равных спин-спиновых взаимодействий, ТM $\Phi, 2005$, том 145, номер 3, 411-419

DOI: https://doi.org/10.4213/tmf1909

Использование Общероссийского математического портала Math-Net.Ru подразумевает, что вы прочитали и согласны с пользовательским соглашением

http: //www . mathnet.ru/rus/agreement

Параметры загрузки:

IP : 18.234 .197 .8

26 апреля 2023 г., 16:51:15 


\section{НЕКОТОРЫЕ ТОЧНЫЕ РЕШЕНИЯ ДЛЯ МОДЕЛИ РАВНЫХ СПИН-СПИНОВЫХ ВЗАИМОДЕЙСТВИЙ}

Для модели равных спин-спиновых взаимодействий получены точные гейзенберговские выражения для компонент оператора спина, с помощью которых найдены соотношения для спиновых операторов под знаком термодинамического среднего. Полученные точные соотношения для средних позволяют описать термодинамику рассматриваемой модели.

Ключевые слова: спин-спиновые взаимодействия, уравнения движения Гейзенберга, термодинамика.

\section{1. ВВЕДЕНИЕ}

Пристальное внимание исследователей к спин-спиновому взаимодействию (CСВ) насчитывает к настоящему моменту несколько десятилетий благодаря тому, что оно определяет тонкие особенности динамики и кинетики электронных и ядерных парамагнитных спин-систем. Гамильтониан парамагнетика (электронного и ядерного) имеет вид [1]

$$
\begin{gathered}
H=H_{z}+H_{d}+H_{d d}, \\
H_{z}=-\sum_{f} h_{f} S_{f}^{z}, \quad H_{d}=-\frac{1}{2} \sum_{f, g} A_{f, g} S_{f}^{z} S_{g}^{z}, \\
H_{d d}=-\frac{1}{4} \sum_{f, g} B_{f, g}\left(S_{g}^{-} S_{f}^{+}+S_{g}^{+} S_{f}^{-}\right) .
\end{gathered}
$$

Здесь

$$
\begin{gathered}
S^{ \pm}=S^{x} \pm i S^{y}, \quad A_{f, g}=V_{f, g}+J_{f, g} \\
V_{f, g}=\frac{\hbar^{2} \gamma^{2}}{R_{f, g}^{3}}\left(1-3 \cos \theta_{f, g}\right), \quad B_{f, g}=-A_{f, g}+J_{f, g},
\end{gathered}
$$

${ }^{*}$ Казанский физико-технический институт КазНЦ РАН, Казань, Россия

${ }^{\dagger}$ Казанский государственный университет, Казань, Россия. E-mail: nigmat@knet.ru

${ }^{\ddagger}$ Казанский государственный энергетический университет, Казань, Россия 
где $S_{f}^{\alpha}-\alpha$-компонента спина $S_{f}, \quad \alpha=x, y, z$, расположенного в точке $f$; $\left(R_{f, g}, \theta_{f, g}, \varphi_{f, g}\right)$ - сферические координаты вектора, соединяющего точки расположения спинов $f$ и $g$ в лабораторной системе координат с осью $z$, параллельной постоянному магнитному полю $H_{0} ; \gamma$ - гиромагнитное отношение; $\omega_{0}=\gamma H_{0}$ - зееманова частота; $J_{f, g}$ - обменный интеграл. В выражении (1) приведена только секулярная часть спин-спинового и обменного взаимодействий, которая в силу коммутации с основным гамильтонианом $H_{z}$ вносит существенно больший вклад в динамику и кинетику спин-систем, чем остальные части ССВ [2]. ССВ представляют собой типичньй пример многочастичных взаимодействий, поэтому не приходится говорить о точной диагонализации гамильтониана (1) в случае, если число взаимодействуюших спинов $N>10$. Даже если будет получено численное решение для подобного коллектива магнитных частиц, окажется весьма затруднительно распорядиться этими сведениями, так как число состояний $M=2^{N}$ экспоненциально растет с ростом $N$. Поэтому сушествуюшие теории вынуждены ограничиться макроскопичекими характеристиками СCВ - временем спин-спиновой релаксации, вторым и четвертым моментами резонансных линий и т.д. [1]

Недавно при изучении ядерного магнитного резонанса (ЯМР) протонов в молекулах нематического жидкого кристалла [3] был экспериментально продемонстрирован принципиально новый спектр ЯМР, состояший из множества узких хорошо разрешенных резонансных линий на месте обычно наблюдаемой единой уширенной ССВ резонансной линии. В настоящее время число узких линий доведено до 1024 [4]. Этот результат открьвает новые возможности в изучении динамики и кинетики дипольно-связанных спиновых систем, и, по мнению авторов работ [3], [4], представляет новую перспективную физическую среду для квантовой информатики.

Таким образом, теоретическое описание квантово-механических свойств дипольносвязанных спиновых систем является чрезвычайно актуальной проблемой.

Жидкий кристалл, в котором наблюдался спин-спиновый мультиплетный спектр с узкими линиями [3], состоит из частично ориентированных молекул, каждая из которых содержит $N=19$ взаимодействующих протонов, а межмолекулярное ССВ пренебрежимо мало. Речь идет, таким образом, об определении более полумиллиона квантовых состояний, т.е. о совершенно бесперспективной для точного решения задаче. Кроме того, необходимо отметить, что при определенных значениях углов $\theta_{f, g}$ взаимодействие между близлежашими спинами может оказаться меньше, чем между отдаленными. К тому же сама ориентация спинов в пространстве зафиксирована неточно, и в качестве констант взаимодействия фигурируют не подлинные значения, а их усредненная тепловым движением часть. Это усреднение ССВ для протонов, расположенных в разных частях молекулы, не одинаково: в более подвижных фрагментах эффект усреднения значительнее. Следовательно, продвижение возможно лишш с помощью теоретической модели, упрощающей задачу, но сохраняюшей при этом ее основные черты. По нашему мнению, такой моделью может служить модель равных спин-спиновых взаимодействий (РССВ) с гамильтонианом

$$
\begin{gathered}
H^{*}=H_{z}+H_{d}^{*}+H_{d d}^{*}, \\
H_{z}=-\omega_{0} \sum_{f} S_{f}^{z}, \quad H_{d}^{*}=-\frac{A}{2} \sum_{f, f^{\prime}} S_{f}^{z} S_{f^{\prime}}^{z}, \quad H_{d d}^{*}=-\frac{B}{4} \sum_{f, f^{\prime}}\left(S_{f}^{+} S_{f^{\prime}}^{-}+S_{f^{\prime}}^{-} S_{f}^{+}\right),
\end{gathered}
$$

где $A$ и $B$ суть средние значения параметров $A_{f f^{\prime}}$ и $B_{f f^{\prime}}$ для молекулы. Этот гамильто- 
ниан применялся ранее для исследования проблем неэргодической динамики на примере равносильно взаимодействуюших спинов $1 / 2$ [5]. Нам представляется, что этот гамильтониан может найти более широкое применение: он может использоваться для описания свойств различных спиновых систем в мезоскопических магнитоактивных кластерах.

Усредненные константы описывают взаимодействие выбранного спина со всеми остальными, сохраняя различия между продольными $(A)$ и поперечными $(B)$ спиновыми компонентами. Эти константы могут быть получены из реального потенциала взаимодействия как

$$
\langle A\rangle=\frac{1}{N} \sum_{f, f^{\prime}} A_{f, f^{\prime}}, \quad \Delta A=\sqrt{\frac{1}{N} \sum_{f, f^{\prime}}\left(A_{f, f^{\prime}}-A\right)^{2}}, \quad A=\langle A\rangle \pm \Delta A ;
$$

то же для константы $B$.

В настояшей работе для модели РССВ мы получим точные выражения для компонент оператора спина в представлении Гейзенберга, с помощью которых определим точные соотношения для средних от спиновых операторов, позволяюшие рассчитывать термодинамические величины рассматриваемой модели.

\section{2. ОСНОВНЫЕ УРАВНЕНИЯ МОДЕЛИ}

Перейдем в представление Гейзенберга и запишем уравнения движения для компонент оператора спина $S_{f}$ :

$$
\begin{aligned}
\frac{d S_{f}^{+}}{d t} & =i\left[H^{*}, S_{f}^{+}\right]=-i\left(\omega_{0}+A \sigma_{f}^{z}\right) S_{f}^{+}+i B \sigma_{f}^{+} S_{f}^{z}= \\
& =-i\left(\omega_{0}+A S^{z}-\frac{A+B}{2}\right) S_{f}^{+}+i B S^{+} S_{f}^{z} \\
\frac{d S_{f}^{-}}{d t} & =i\left[H^{*}, S_{f}^{-}\right]=i\left(\omega_{0}+A \sigma_{f}^{z}\right) S_{f}^{-}-i B \sigma_{f}^{-} S_{f}^{z}= \\
& =i\left(\omega_{0}+A S^{z}-\frac{A+B}{2}\right) S_{f}^{-}-i B S^{-} S_{f}^{z} ; \\
\frac{d S_{f}^{z}}{d t} & =i\left[H^{*}, S_{f}^{z}\right]=\frac{i B}{2}\left(\sigma_{f}^{-} S_{f}^{+}-\sigma_{f}^{+} S_{f}^{-}\right)=\frac{i B}{2}\left(S^{-} S_{f}^{+}-S^{+} S_{f}^{-}\right)+i B S_{f}^{z} .
\end{aligned}
$$

Здесь через $\sigma_{f}^{z}, \sigma_{f}^{ \pm}$обозначены операторы

$$
\sigma_{f}^{z}=\sum_{f^{\prime} \neq f} S_{f^{\prime}}^{z}=S^{z}-S_{f}^{z}, \quad \sigma_{f}^{ \pm}=\sum_{f^{\prime} \neq f} S_{f^{\prime}}^{ \pm}=S^{ \pm}-S_{f}^{z}
$$

где $S^{z}, S^{ \pm}$- компоненты оператора полного спина $S$ рассматриваемой спиновой системы. Просуммировав уравнения (3) по узлу $f$, найдем уравнения движения для компонент полного спина:

$$
\begin{gathered}
\frac{d S^{+}}{d t}=-i\left(\omega_{0}+(A-B)\left(S^{z}-\frac{1}{2}\right)\right) S^{+}, \\
\frac{d S^{-}}{d t}=i\left(\omega_{0}+(A-B)\left(S^{z}+\frac{1}{2}\right)\right) S^{-}, \\
\frac{d S^{z}}{d t}=0 .
\end{gathered}
$$


Отсюда легко получим зависимости компонент оператора полного спина от времени:

$$
\begin{gathered}
S^{ \pm}(t)=e^{\mp i \theta_{ \pm} t} S^{ \pm}, \quad \theta_{ \pm}=\omega_{0}+(A-B)\left(S^{z} \mp \frac{1}{2}\right) \\
S^{z}(t)=S^{z} .
\end{gathered}
$$

Оператор $S^{z}$, как это и должно быть, является интегралом движения. В следуюшем разделе мы получим дифференциальные уравнения для определения $S_{f}^{z}(t), S_{f}^{+}(t)$ с коэффициентами, являющимися интегралами движения, и проинтегрируем их.

\section{3. РАСЧЕТ ЗАВИСИМОСТЕЙ $S_{f}^{z}(t), S_{f}^{+}(t)$}

Продифференцируем уравнение (3) по времени и, проведя некоторые несложные манипуляции со спиновыми операторами, а также используя уравнения (5), получим

$$
\frac{d^{2} S_{f}^{z}}{d t^{2}}=B^{2}\left(S^{z} Q_{f}-\left(S^{2}-\left(S^{z}\right)^{2}\right) S_{f}^{z}\right)+i B \frac{d S_{f}^{z}}{d t},
$$

где через $Q_{f}$ обозначен оператор

$$
Q_{f}=\frac{S^{-} S_{f}^{+}+S^{+} S_{f}^{-}}{2}
$$

а $S^{2}$ - квадрат оператора полного спина, которьй определяется следуюшим выражениem:

$$
S^{2}=\left(S^{z}\right)^{2}+\frac{S^{+} S^{-}+S^{-} S^{+}}{2}=\left(S^{z}\right)^{2}+S^{+} S^{-}-S^{z} .
$$

Далее продифференцируем уравнение (8) еше раз по времени и получим уравнение

$$
\frac{d^{3} S_{f}^{z}}{d t^{3}}=B^{2}\left(S^{z} \frac{d Q_{f}}{d t}-\left(S^{2}-\left(S^{z}\right)^{2}\right) \frac{d S_{f}^{z}}{d t}\right)+i B \frac{d^{2} S_{f}^{z}}{d t^{2}}
$$

Используя выражение (9) и уравнения (3), (5), найдем выражение для $d Q_{f} / d t$, входящее в уравнение (11):

$$
\frac{d Q_{f}}{d t}=-S^{z} \frac{d S_{f}^{z}}{d t}
$$

Подставляя соотношение (12) в уравнение (11), окончательно получим дифференциальное уравнение для определения $S_{f}^{z}(t)$ :

$$
\frac{d^{3} S_{f}^{z}}{d t^{3}}-i B \frac{d^{2} S_{f}^{z}}{d t^{2}}+B^{2} S^{2} \frac{d S_{f}^{z}}{d t}=0
$$

Данное уравнение примечательно тем, что его коэффициенты не зависят от времени и коммутируют с оператором $S_{f}^{z}$. Поэтому это операторное дифференциальное уравнение может быть легко проинтегрировано, и мы получим следуюшее выражение для зависимости $S_{f}^{z}(t)$ :

$$
S_{f}^{z}(t)=\left(e^{i B(1 / 2+\Omega) t}-1\right) C_{+}^{z}+\left(e^{i B(1 / 2-\Omega) t}-1\right) C_{-}^{z}+S_{f}^{z},
$$


где $C_{ \pm}^{z}$ - операторы констант интегрирования, уравнения для определения которых имеют вид

$$
\begin{aligned}
& \dot{S}_{f}^{z}=i B\left(\left(\frac{1}{2}+\Omega\right) C_{+}^{z}+\left(\frac{1}{2}-\Omega\right) C_{-}^{z}\right) \\
& \ddot{S}_{f}^{z}=-B^{2}\left(\left(\frac{1}{2}+\Omega\right)^{2} C_{+}^{z}+\left(\frac{1}{2}-\Omega\right)^{2} C_{-}^{z}\right) .
\end{aligned}
$$

Здесь $\Omega=\sqrt{1 / 4+S^{2}}$. Используя уравнения (3) и (8), найдем выражения для $\dot{S}_{f}^{z}, \ddot{S}_{f}^{z}$ :

$$
\begin{gathered}
\dot{S}_{f}^{z}=\frac{i B}{2}\left(S^{-} S_{f}^{+}-S^{+} S_{f}^{-}\right)+i B S_{f}^{z} \\
\ddot{S}_{f}^{z}=\frac{B^{2}}{2}\left(S^{z}-1\right) S^{-} S_{f}^{+}+\frac{B^{2}}{2}\left(S^{z}+1\right) S^{+} S_{f}^{-}-B^{2}\left(S^{2}-\left(S^{z}\right)^{2}+1\right) S_{f}^{z}
\end{gathered}
$$

Подставляя эти соотношения в уравнения (15), получим выражения для операторов констант интегрирования:

$$
\begin{aligned}
& C_{+}^{z}=\frac{S^{2} K_{z}+(1 / 2-\Omega) L_{z}}{2 S^{2} \Omega} \\
& C_{-}^{z}=-\frac{S^{2} K_{z}+(1 / 2+\Omega) L_{z}}{2 S^{2} \Omega}
\end{aligned}
$$

где

$$
\begin{aligned}
K_{z} & =S^{z} \frac{\left(S^{+} S_{f}^{-}+S^{-} S_{f}^{+}\right)}{2}-\left(S^{2}-\left(S^{z}\right)^{2}\right) S_{f}^{z}, \\
L_{z} & =S_{f}^{z}+\frac{S^{-} S_{f}^{+}-S^{+} S_{f}^{-}}{2} .
\end{aligned}
$$

С учетом (18) выражение для $S_{f}^{z}(t)$ перепишется в виде

$$
S_{f}^{z}(t)=\Phi_{1}\left(S^{2}, t\right) K_{z}+\Phi_{2}\left(S^{2}, t\right) L_{z}+S_{f}^{z}
$$

где функции $\Phi_{1}\left(S^{2}, t\right), \Phi_{2}\left(S^{2}, t\right)$ определяются как

$$
\begin{aligned}
\Phi_{1}\left(S^{2}, t\right) & =\frac{\left(e^{i B(1 / 2+\Omega) t}-1\right)(1 / 2-\Omega)-\left(e^{i B(1 / 2-\Omega) t}-1\right)(1 / 2+\Omega)}{2 S^{2} \Omega}= \\
& =\frac{i e^{i B t / 2}(\sin (B \Omega t)-2 \Omega \cos (B \Omega t))+2 \Omega}{2 S^{2} \Omega}, \\
\Phi_{2}\left(S^{2}, t\right) & =\frac{e^{i B(1 / 2+\Omega) t}-e^{i B(1 / 2-\Omega) t}}{2 \Omega}=\frac{i e^{i B t / 2} \sin (B \Omega t)}{\Omega} .
\end{aligned}
$$

Далее перейдем к выводу дифференциального уравнения для определения $S_{f}^{+}(t)$. Продифференцируем первое из уравнений (3) по времени, а затем, используя коммутационные соотношения для спиновых операторов и уравнения $(3),(5)$, получим

$$
\frac{d^{2} S_{f}^{+}}{d t^{2}}=-i\left(2 \theta_{+}+B\left(S^{z}-2\right)\right) \frac{d S_{f}^{+}}{d t}+\left(\theta_{+}^{2}+B \theta_{+}\left(S^{z}-2\right)-B^{2}\left(S^{2}-\left(S^{z}-1\right)^{2}\right)\right) S_{f}^{+}+B^{2} S^{+} Q_{f} .
$$


Дифференцируя уравнение (23) по времени и используя уравнения (5), (23), окончательно найдем дифференциальное уравнение для $S_{f}^{+}(t)$ :

$\frac{d^{3} S_{f}^{+}}{d t^{3}}+i\left(3 \theta_{+}-B\right) \frac{d^{2} S_{f}^{+}}{d t^{2}}+\left(B^{2} S^{2}-\theta_{+}\left(3 \theta_{+}-2 B\right)\right) \frac{d S_{f}^{+}}{d t}+i \theta_{+}\left(B^{2} S^{2}-\theta_{+}\left(\theta_{+}-B\right)\right) S_{f}^{+}=0$.

Мы снова получили операторное дифференциальное уравнение с не зависяшими от времени коэффициентами. На первый взгляд может показаться, что уравнение (24) достаточно сложное, но если произвести переход от оператора $S_{f}^{+}(t)$ к оператору $\widetilde{S}_{f}^{+}(t)$ по формуле

$$
S_{f}^{+}(t)=e^{-i \theta_{+} t} \widetilde{S}_{f}^{+}(t)
$$

то уравнение (24) преобразуется к виду

$$
\frac{d^{3} \widetilde{S}_{f}^{+}}{d t^{3}}-i B \frac{d^{2} \widetilde{S}_{f}^{+}}{d t^{2}}+B^{2} S^{2} \frac{d \widetilde{S}_{f}^{+}}{d t}=0
$$

Уравнение (26) совпадает по виду с уравнением $(13)$ для $S_{f}^{z}(t)$ и легко интегрируется. В результате решение уравнения (24) с учетом (25) запишется как

$$
S_{f}^{+}(t)=e^{-i \theta_{+} t}\left\{\left(e^{i B(1 / 2+\Omega) t}-1\right) C_{+}^{+}+\left(e^{i B(1 / 2-\Omega) t}-1\right) C_{-}^{+}+S_{f}^{+}\right\},
$$

где $C_{ \pm}^{+}$- операторы констант интегрирования, уравнения для определения которых имеют вид

$$
\begin{aligned}
i B\left(\left(\frac{1}{2}+\Omega\right) C_{+}^{+}+\left(\frac{1}{2}-\Omega\right) C_{-}^{+}\right) & =i \theta_{+} S_{f}^{+}+S_{f}^{+} \\
-B^{2}\left(\left(\frac{1}{2}+\Omega\right)^{2} C_{+}^{+}+\left(\frac{1}{2}-\Omega\right)^{2} C_{-}^{+}\right) & =-\theta_{+}^{2} S_{f}^{+}+2 i \theta_{+} S_{f}^{+}+S_{f}^{+} .
\end{aligned}
$$

Используя уравнения (3) и (23), найдем выражения для правых частей уравнений (28):

$$
\begin{aligned}
\dot{S}_{f}^{+}+i \theta_{+} S_{f}^{+}= & -i B\left(S^{z}-1\right) S_{f}^{+}+i B S^{+} S_{f}^{z} \\
\ddot{S}_{f}^{+}+2 i \theta_{+} \dot{S}_{f}^{+}-\theta_{+}^{2} S_{f}^{+}= & -B^{2}\left(S^{2}-S^{z}+1\right) S_{f}^{+}+ \\
& +B^{2}\left(S^{z}-2\right) S^{+} S_{f}^{z}+\frac{B^{2}}{2} S^{+}\left(S^{+} S_{f}^{-}+S^{-} S_{f}^{+}\right) .
\end{aligned}
$$

Подставляя выражения (29), (30) в уравнения (28), получим окончательные выражения для констант интегрирования:

$$
\begin{aligned}
& C_{+}^{+}=\frac{S^{2} K_{+}+(1 / 2-\Omega) L_{+}}{2 S^{2} \Omega}, \\
& C_{-}^{+}=-\frac{S^{2} K_{+}+(1 / 2+\Omega) L_{+}}{2 S^{2} \Omega},
\end{aligned}
$$

где

$$
\begin{aligned}
K_{+} & =S^{+} \frac{S^{+} S_{f}^{-}+S^{-} S_{f}^{+}}{2}+\left(S^{z}-1\right) S^{+} S_{f}^{z}-S^{2} S_{f}^{+}, \\
L_{+} & =S^{+} S_{f}^{z}-\left(S^{z}-1\right) S_{f}^{+} .
\end{aligned}
$$


Принимая во внимание (31), запишем выражение (27) для $S_{f}^{+}(t)$ в виде

$$
S_{f}^{+}(t)=e^{-i \theta_{+} t}\left\{\Phi_{1}\left(S^{2}, t\right) K_{+}+\Phi_{2}\left(S^{2}, t\right) L_{+}+S_{f}^{+}\right\}
$$

где функции $\Phi_{1}\left(S^{2}, t\right), \Phi_{2}\left(S^{2}, t\right)$ определяются выражениями $(21)$ и (22).

В следуюшем разделе мы получим соотношения для средних от спиновых операторов, которыепозволяют определять термодинамические средние и корреляционные функции рассматриваемой модели.

\section{4. ВЫВОД ОПЕРАТОРНЫХ СООТНОШЕНИЙ, ОПРЕДЕЛЯЮШИХ ТЕРМОДИНАМИЧЕСКИЕ СРЕДНИЕ МОДЕЛИ}

Расчеты, проводимые в данном разделе, будут базироваться на следующем операторном соотношении под знаком среднего:

$$
\langle B(-i \beta) C\rangle=\langle C B\rangle,
$$

где $B(t)$ - произвольньй квантово-механический оператор в представлении Гейзенберга, $C$ - произвольный квантово-механический оператор в представлении Шредингера. Уравнение (35) следует из свойства циклической перестановки операторов под знаком $\mathrm{Sp}$. Соотношение (35) удобно тем, что позволяет непосредственно от операторов в представлении Гейзенберга перейти к выражениям для равновесных термодинамических средних операторов.

Из уравнения (35) следует, что нам необходимо знание выражений для $S_{f}^{z}(-i \beta)$, $S_{f}^{+}(-i \beta)$, которые можно легко получить из выражений $(20),(34)$, положив $t=-i \beta$ :

$$
\begin{aligned}
S_{f}^{z}(-i \beta) & =\Phi_{1}\left(S^{2}, \beta\right) K_{z}+\Phi_{2}\left(S^{2}, \beta\right) L_{z}+S_{f}^{z}, \\
S_{f}^{+}(-i \beta) & =e^{-\beta \theta_{+}}\left\{S_{f}^{+}+\Phi_{1}\left(S^{2}, \beta\right) K_{+}+\Phi_{2}\left(S^{2}, \beta\right) L_{+}\right\},
\end{aligned}
$$

где

$$
\begin{aligned}
& \Phi_{1}\left(S^{2}, \beta\right) \equiv \Phi_{1}\left(S^{2},-i \beta\right)=\frac{e^{\beta B / 2}(i \operatorname{sh}(\beta B \Omega)-2 \Omega \operatorname{ch}(\beta B \Omega))+2 \Omega}{2 S^{2} \Omega} \\
& \Phi_{2}\left(S^{2}, \beta\right) \equiv \Phi_{2}\left(S^{2},-i \beta\right)=\frac{e^{\beta B / 2} \operatorname{sh}(\beta B \Omega)}{\Omega} .
\end{aligned}
$$

Далее перейдем к непосредственному определению соотношений для термодинамичеких средних. Полагая в уравнении (35) $B(-i \beta)=e^{\beta \theta_{+}} S_{f}^{+}(-i \beta), C=S_{f}^{-} A$, где $A-$ произвольный квантово-механический оператор, коммутируюший с операторами $B$ и $S_{f}^{-}$, получим

$$
\left\langle e^{\beta \theta_{+}} S_{f}^{+}(-i \beta) S_{f}^{-}\right\rangle=\left\langle\Phi_{1}\left(S^{2}, \beta\right) K_{+} S_{f}^{-}+\Phi_{2}\left(S^{2}, \beta\right) L_{+} S_{f}^{-}+S_{f}^{+} S_{f}^{-}\right\rangle=\left\langle S_{f}^{-} e^{\beta \theta_{+}} S_{f}^{+}\right\rangle .
$$

5 Теоретическая и математическая физика, т. 145, № 3,2005 г. 
Чтобы привести это уравнение к компактному виду, нам понадобится знание коммутатора операторов $S_{f}^{+}$и $e^{\beta \theta_{+}}$, расчет которого приведен в приложении. Принимая во внимание коммутационное соотношение (П.8) и соотношения $S_{f}^{+} S_{f}^{-}=1 / 2+S_{f}^{z}, S_{f}^{-} S_{f}^{+}=1 / 2-$ $S_{f}^{z}$, уравнение (40) перепишем в виде

$$
\left\langle\Phi_{1}\left(S^{2}, \beta\right) K_{+} S_{f}^{-}+\Phi_{2}\left(S^{2}, \beta\right) L_{+} S_{f}^{-}+S_{f}^{+} S_{f}^{-}+\frac{1}{2}+S_{f}^{z}\right\rangle=\left\langle\left(\frac{1}{2}-S_{f}^{z}\right) e^{\beta \theta_{-}}\right\rangle .
$$

Далее, учитывая выражения $(32),(33)$ для $K_{+}, L_{+}$, найдем

$$
\begin{aligned}
K_{+} S_{f}^{-} & =-\frac{1}{2}\left(\left(S^{2}+S^{z}\left(S^{z}-1\right)\right)\left(\frac{1}{2}+S_{f}^{z}\right)+\left(S^{z}-1\right) S^{+} S_{f}^{-}\right), \\
L_{+} S_{f}^{-} & =-\left(\left(S^{z}-1\right)\left(\frac{1}{2}+S_{f}^{z}\right)+\frac{1}{2} S^{+} S_{f}^{-}\right) .
\end{aligned}
$$

Подставляя выражения (42), (43) в уравнение (41), получим

$$
\begin{gathered}
\left\langle\left(\frac{1}{2} \Phi_{1}\left(S^{2}, \beta\right)\left(S^{2}+\left(S^{z}\right)^{2}-S^{z}\right)+\Phi_{2}\left(S^{2}, \beta\right)\left(S^{z}-1\right)-1-e^{\beta \theta_{-}}\right)\left(\frac{1}{2}+S_{f}^{z}\right) A\right\rangle+ \\
+\frac{1}{2}\left\langle\left(\Phi_{1}\left(S^{2}, \beta\right)\left(S^{z}-1\right)+\Phi_{2}\left(S^{2}, \beta\right)\right) S^{+} S_{f}^{-} A\right\rangle+\left\langle e^{\beta \theta_{-}} A\right\rangle=0 .
\end{gathered}
$$

Уравнение (44) представляет собой точное операторное соотношение, связываюшее термодинамические средние спиновых операторов модели РССВ. Получим еше одно подобное соотношение. Полагая в (35) $B(-i \beta)=S_{f}^{z}(-i \beta), C=S_{f}^{z} A$, получим

$$
\left\langle S_{f}^{z}(-i \beta) S_{f}^{z} A\right\rangle=\left\langle S_{f}^{z} S_{f}^{z} A\right\rangle=\frac{1}{4}\langle A\rangle .
$$

Отсюда, принимая во внимание выражение (36), найдем

$$
\begin{aligned}
& \left\langle\left(\Phi_{1}\left(S^{2}, \beta\right) S^{z}-\Phi_{2}\left(S^{2}, \beta\right) S^{+} S_{f}^{-} A\right\rangle-\left\langle\left(\Phi_{1}\left(S^{2}, \beta\right) S^{z}+\Phi_{2}\left(S^{2}, \beta\right) S^{-} S_{f}^{+} A\right\rangle=\right.\right. \\
& \quad=\left\langle\left(\Phi_{1}\left(S^{2}, \beta\right)\left(S^{2}-\left(S^{z}\right)^{2}\right)-\Phi_{2}\left(S^{2}, \beta\right) A\right\rangle .\right.
\end{aligned}
$$

Операторные соотношения (44) и (46) представляют собой аналог так называемого уравнения Келлена [6] для модели Изинга и позволяют определить необходимые термодинамические функции модели в рамках определенного приближения. Точное решение этих уравнений не представляется возможным.

\section{5. ЗАКЛЮЧЕНИЕ}

Для модели РССВ путем повторного дифференцирования из системы гейзенберговских уравнений движения для спиновых операторов удается получить систему несвязанных интегрируемых дифференциальных уравнений третьего порядка для нахождения гейзенберговсих выражений для компонент оператора спина. Явные гейзенберговские выражения компонент оператора спина позволяют, во-первых, исследовать спиновую динамику систем, описываемых данной моделью, включая магнитньй резонанс, а во-вторых, описать термодинамику модели. Операторные уравнения (44), (46), полученные здесь, к сожалению, не допускают точного решения, поэтому для нахождения термодинамических величин модели возникает необходимость выбора приближения. В данной работе авторы решили не загромождать полученные здесь оригинальные результаты расчетом приближенных термодинамических средних модели. Это будет являться целью следующей работы. 
ПРИЛОЖЕНИЕ

Определим коммутационное соотношение для операторов $S_{f}^{+}$и $e^{\beta \theta_{+}}$. Для этого воспользуемся так называемым тождеством Кубо [7]

$$
\left[U, e^{-\beta V}\right]=-e^{-\beta L} \int_{0}^{\beta} e^{\lambda L}[U, L] e^{-\lambda V} d \lambda,
$$

где $U, V$ - произвольные операторы. Полагая $U=S_{f}^{+}, V=-\theta_{+}$, получим

$$
\left[S_{f}^{+}, e^{\beta \theta_{+}}\right]=e^{\beta \theta_{+}} \int_{0}^{\beta} e^{-\lambda \theta_{+}}\left[S_{f}^{+}, \theta_{+}\right] e^{\lambda \theta_{+}} d \lambda .
$$

Далее, принимая во внимание выражение для $\theta_{+}(6)$, найдем выражение для коммутатора $\left[S_{f}^{+}, \theta_{+}\right]$:

$$
\left[S_{f}^{+}, \theta_{+}\right]=(A-B)\left[S_{f}^{+}, S^{z}\right]=-(A-B) S_{f}^{+} .
$$

Подставляя выражение (П.3) в соотношение (П.2), получим

$$
\left[S_{f}^{+}, e^{\beta \theta_{+}}\right]=-(A-B) e^{\beta \theta_{+}} \int_{0}^{\beta} e^{-\lambda \theta_{+}} S_{f}^{+} e^{\lambda \theta_{+}} d \lambda .
$$

Для расчета подынтегрального выражения в (П.4) воспользуемся формулой

$$
e^{B} A e^{-B}=A+[B, A]+\frac{1}{2 !}[B,[B, A]]+\frac{1}{3 !}[B,[B,[B, A]]]+\cdots,
$$

с учетом которой можно записать

$$
e^{-\lambda \theta_{+}} S_{f}^{+} e^{\lambda \theta_{+}}=e^{-\lambda(A-B)} S_{f}^{+}
$$

Подставляя выражение (П.6) в соотношение (П.4), окончательно найдем выражение для коммутатора операторов $S_{f}^{+}$и $e^{\beta \theta_{+}}$:

$$
\left[S_{f}^{+}, e^{\beta \theta_{+}}\right]=e^{\beta \theta_{+}}\left(e^{-\beta(A-B)}-1\right) S_{f}^{+} .
$$

Отсюда непосредственно следует следуюшее правило коммутации операторов $S_{f}^{+}$ и $e^{\beta \theta_{+}}$:

$$
e^{\beta \theta_{+}} S_{f}^{+}=S_{f}^{+} e^{\beta \theta_{-}} .
$$

\section{Список литературы}

[1] А. Абрагам. Ядерный магнетизм. М.: ИИЛ, 1963.

[2] В. А. Ацаркин. Динамическая поляризация ядер в твердых диэлектриках. М.: Наука, 1980; В. А. Ацаркин, М.И. Родак. УфН. 1972. Т. 107. № 1. С. 3-27; М. Гольдман. Спиновая температура и ЯМР в твердых телах. М.: Мир, 1972.

[3] A. K. Khitrin, V. L. Ermakov, B. M. Fung. Chem. Phys. Lett. 2002. V. 350. № 1-2. P. 161-166.

[4] A. K. Khitrin, V. L. Ermakov, B. M. Fung. J. Chem. Phys. 2002. V. 117. № 15. P. 6903-6906.

[5] М. Г. Рудавец, Э. Б. Фельдман. Письма в ЖЭТФ. 2002. Т. 75. № 12. С. 760-762.

[6] H. B. Callen. Phys. Rev. 1963. V. 22. P. 123-129.

[7] Д. Н. Зубарев. Неравновесная статистическая механика. М.: Наука, 1971.

Поступила в редакцию 21.IV.2005 г. 